\title{
Enfermedades congénitas de la coagulación y manejo en Odontopediatría
}

\section{Resumen}

El revisar un paciente en odontopediatría con hematomas y hemorragias es frecuente. El diagnóstico y el tratamiento dependen del conocimiento de los mecanismos normales de la hemostasia y de los laboratorios que los analizan ${ }^{1}$. Las manipulaciones en la cavidad bucal, máxime aquellas con extravasación de sangre, son un riesgo para los pacientes con trastornos de la coagulación, la prevención es la forma de evitar complicaciones, para ello debe disponer: de una historia clínica con énfasis en antecedentes personales y familiares de problemas hemorrágicos y del conoci-
Juan Manuel-Cortes-Ramírez ${ }^{1}$

Juan Manuel de Jesús-Cortes-de la Torre ${ }^{2}$

Raúl Arturo-Cortes-de la Torre ${ }^{3}$

Carmen de la Luz-Ayala-Escandón ${ }^{4}$

Laura Otilia Salazar Fernández $z^{5}$

Sofía Isabel Carrillo Aguilar ${ }^{6}$

Artigo de revisão

\section{Enfermidades congênitas de coagulação e seu manejo em odontopediatria}

\section{Resumo}

Atender um paciente em odontopediatria com hematomas e hemorragias é frequente. $\mathrm{O}$ diag- miento de las patologías más frecuentes que lo originan así como su manejo ${ }^{2}$. Las hemorragias menores, son por factores locales, las graves por trastornos sistémicos de la hemostasia, como las coagulopatias congénitas², grupo heterogéneo de enfermedades secundarias a alteraciones de las proteínas plasmáticas de la hemostasia primaria (ejemplo factor de von Willebrand), de la coagulación o de la fibrinólisis, defecto de un factor o combinación de 2 o más. Esta revisión se propone responder a estas interrogantes.

Palabras claves: coagulación, hemorragia, hemostasia, odontopediatría.

\footnotetext{
${ }^{1}$ Cardiólogo internista, docente titular C de la unidad académica de medicina humana y ciencias de la salud (doc_juancard52@hotmail.com)

${ }^{2}$ Residente Medicina Interna Hospital de San José Tec. De Monterrey

${ }^{3}$ Médico pasante en servicio social de los SSZ

${ }^{4}$ Docente de la especialidad en Odontopediatria UAZ

${ }^{5}$ Residente de Odontopediatria de la UANL

${ }^{6}$ Alumna de la unidad académica de medicina humana UAZ (sofii_lu@hotmail.com)
} 
aquelas com extravasamento de sangue, são um risco para os pacientes com distúrbios de coagulação. As complicações podem ser prevenidas, para isso deve-se dispor de: uma história clínica com ênfase nos antecedentes pessoais e familiares de distúrbios hemorrágicos e conhecimento sobre as patologias hemorrágicas mais frequentes bem como do seu manejo. As hemorragias menores são por fatores locais e as graves por desordens sistêmicas da hemóstasia, como as coagulopatias congênitas, grupo heterogêneo de doenças secundárias às alterações das proteínas plasmáticas da hemóstasia primária (exemplo fator de von Willebrand), da ou da fibrinólise, defeito de um fator ou combinação de 2 ou mais. Esta revisão propõe-se a responder a estas questões.

Palavras chaves: coagulação, hemorragia, hemóstasia, odontopediatría.

Review article

\section{Congenital diseases of coagulation and management in Odontopediatria}

\section{Abstract}

Reviewing a patient in Odontopediatria with hematomas and bleeding is common. Diagnosis and treatment depend on knowledge of normal mechanisms of hemostasis and laboratories that analyze. Manipulations in the oral cavity, especially those with extravasation of blood, are a risk for patients with bleeding disorders, prevention is the avoidance of complications, for this reason it must have: a medical history with emphasis on background and family of bleeding problems and knowledge of the most common diseases that originate and handling ${ }^{2}$. Minor hemorrhages, occur due to local factors, the severe systemic disorders of hemostasis, such as congenital coagulopathy, heterogeneous group of diseases secondary to alterations of plasma proteins of primary hemostasis (eg von Willebrand factor) of coagulation or fibrinolysis, are caused by the absence of a factor or combination of 2 or more. This review aims to answer these questions.
Keywords: coagulation, bleeding, hemostasis, dentistry.

\section{Introducción}

La capacidad para controlar el flujo sanguíneo luego de una lesión vascular se logra por: 1 . Hemostasia, la constricción vascular que limita el flujo al área lesionada. 2. La activación plaquetaria que a su vez libera ADP, el eicosanoide, $\mathrm{TXA}_{2^{\prime}}$ que activa más plaquetas, serotonina, fosfolípidos, lipoproteínas y otras proteínas de la cascada de coagulación. La agregación plaquetaria, formando un tapón temporal al unirse al colágeno del recubrimiento epitelial de los vasos. 3. La coagulación convierte el fibrinógeno en fibrina y forma una red tridimensional o coágulo que atrapa a otras proteínas, agua, sales y células sanguíneas. Si tiene solo plaquetas se denomina trombo blanco; si glóbulos rojos, trombo rojo. 4 . La reparación del tejido lesionado. El coágulo se disuelve por la plasmina y el flujo sanguíneo se normaliza al repararse el tejido. 
La coagulación es una cascada de reacciones enzimáticas, intervienen proteínas, iones de $\mathrm{Ca}^{2+}$ y fosfolípidos de membranas, se les denomina "Factor" y se le designa por un número de acuerdo a su descubrimiento. De ellos, siete :precalicreína (factor V), protrombina (Factor II), proconvertina (Factor VII), factor antihemofílico beta (IX), factor Stuart $(\mathrm{X})$, tromboplastina plasmática (XI) y factor Hageman (XII), son zimógenos, proenzimas, sin actividad catalítica, se sintetizan en hígado, se activan al hidrolizarse, y a su vez activan a las siguientes enzimas. Hay factores que requieren vitamina $\mathrm{K}$ para su síntesis hepática: el II (protrombina), VII (proconvertina), IX (antihemofílico beta) y X (Stuart). La cascada tiene tres vías: La intrínseca, la extrínseca y la común (Fig. 1). Las 2 primeras son de iniciación, terminan con la conversión del factor $X$ en Xa (la "a" subíndice " significa "activado"), la común finaliza con la conversión de fibrinógeno en fibrina. La formación del trombo rojo ó coágulo como respuesta a anormalidad en un vaso en ausencia de lesión es resultado de la vía intrínseca, significa poco en condiciones fisiológicas. La coagulación inicia cuando la sangre entra en contacto con una superficie diferente al endotelio vascular: la membrana basal, las fibras colágenas del tejido conectivo, las superficies polianiónicas, materiales orgánicos, celulosa, o no orgánicos, vidrio, caolín o resinas.

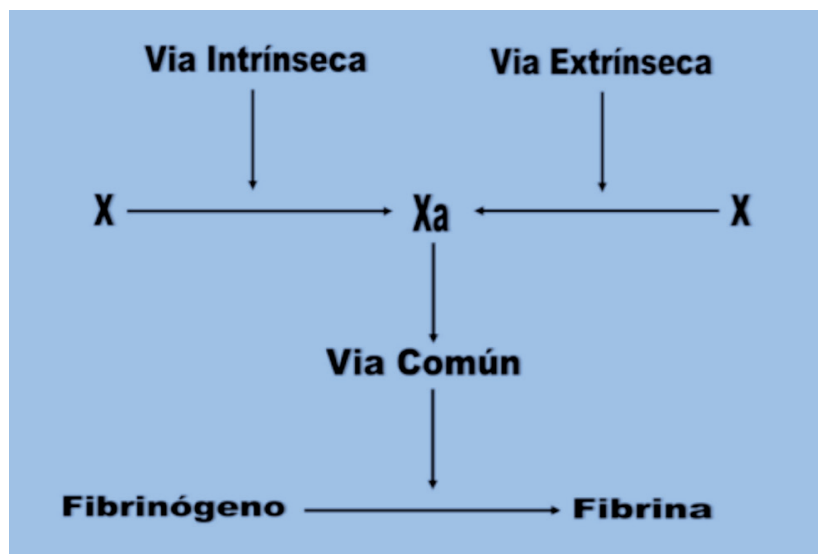

Figura 1. Cascada de la coagulación.

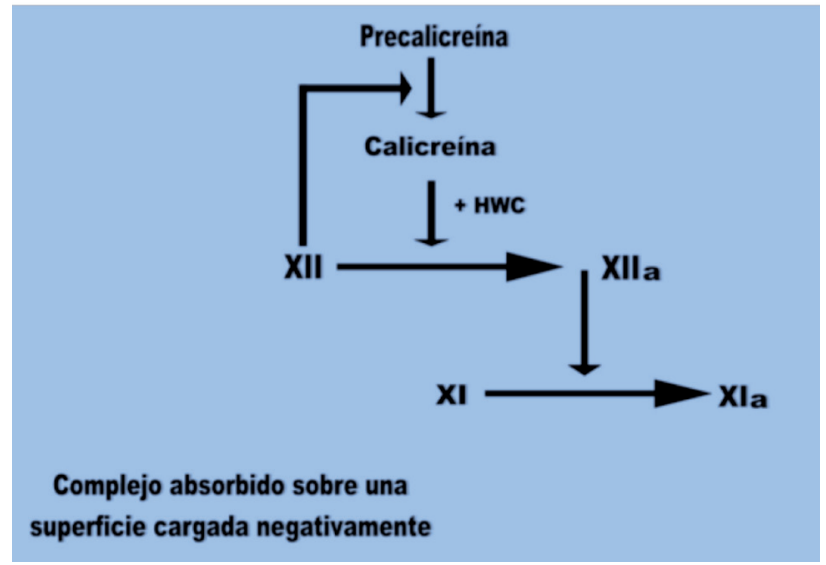

Figura 2. Vía intrínseca.

\section{Vía intrínseca}

1. Formación del factor $X I_{a}$. Participan: Precalicreína (factor V), Quininógeno de alto peso molecular (HMWK) y factores XII y XI, forman el complejo de iniciación. El XII, activa la precalicreína en calicreína y esta convierte el factor XII en XII . La actividad catalítica de calicreína se potencia por el HMWK. La proteasa XII actúa sobre el factor XI para liberar $\mathrm{XI}_{\mathrm{a}}$ (Fig. 2).

2. Formación del factor IX (Fig. 3) El factor IX en presencia de iones $\mathrm{Ca}^{2+}$ y el factor $\mathrm{XI}_{\mathrm{a}}$ liberan al factor IX $\mathrm{I}_{\mathrm{a}}$ El factor IX está ausente en personas con hemofilia tipo B.

3. Formación del factor $X_{a}$. Se forma un complejo sobre las plaquetas por los factores $1 \mathrm{Xa}, \mathrm{X}$, Vlll-Fosfolípidos y $\mathrm{Ca}^{2+}$ actúa sobre el factor

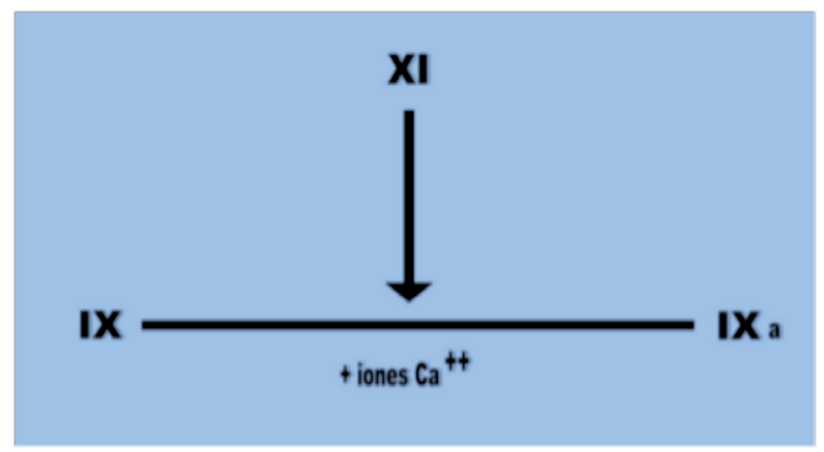

Figura 3. Formación del factor $I X_{a}$. 
$X$ lo convierte en $X_{a}$ y concluye la vía intrínseca. El factor Vlll tiene 2 componentes, el C "componente antihemofílico" cofactor del 1Xa, en la activación del factor X, su ausencia causa la hemofilia A, El R permite la unión del factor V1ll al complejo.

\section{Vía extrínseca}

La sangre al contactar con tejidos lesionados o extractos de tejidos, genera factor $X_{a}$. La activación es mediada por un complejo formado por factor VII, $\mathrm{Ca}^{2+}$ y factor tisular (11l) unido a fosfolípidos de las membranas celulares rotas y de las plaquetas (Fig. 4).

El factor tisular (11l),se sintetiza en el endotelio de los vasos sanguíneos (predomina en pulmón, cerebro y placenta), se encuentra en el interior de las células endoteliales y es secretado en respuesta a una lesión, o por efecto de citoquinas : Factor de Necrosis Tumoral (TNF), InterLeucina 1 (IL-1); o por endotoxinas bacterianas.

1. Formación del factor VII. El factor VII se une al factor tisular utilizando iones $\mathrm{Ca}^{2+}$ como puentes. Este complejo activa al factor VII .

2. Formación del factor $X_{a}$. El complejo VIIa-III$\mathrm{Ca}^{2+}$ convierte el factor $X$ en $X_{a}$. En este punto termina la vía extrínseca y se inicia la vía común.

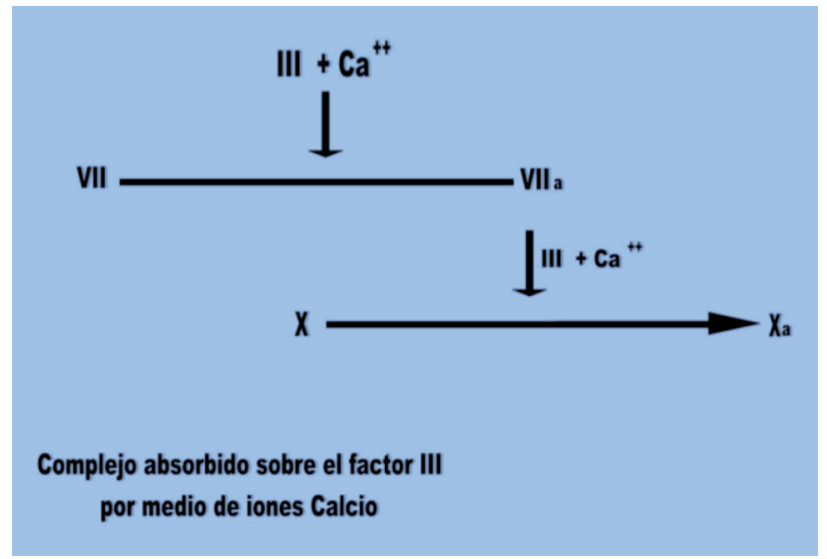

Figura 4. Vía extrínseca.

\section{Vía común}

Al activarse el factor $X$, ambas vías confluyen en la vía común que convierte el fibrinógeno en fibrina, y estabiliza el coágulo e implica tres etapas:

1. Formación de trombina. La trombina (factor II $_{\mathrm{a}}$ ) proviene de la hidroxilación de la protrombina (factor II) por acción del factor $X_{a}$. Se acelera por la formación de un complejo el factor $\mathrm{V}_{\mathrm{a}}$ y $\mathrm{Ca}^{2+}$ sobre la superficie de las plaquetas, va acelerándose a medida que progresa (reacción autoacelerada).

2. Formación de fibrina. Cada mitad del fibrinógeno (factor I) está formada por tres cadenas (A-alfa, B-beta y gamma) que se enrollan. Los extremos de las seis cadenas se reúnen en el centro. La trombina separa cuatro péptidos "fibrinopéptidos" que al eliminarse desaparecen las fuerzas de repulsión intermoleculares, y los monómeros de fibrina forman asociaciones ordenadas en forma de hebras que forman manojos.

3. Entrecruzamiento de la fibrina. Los haces paralelos de fibrina forman enlaces covalentes entre hebras vecinas, "puentes", catalizada por el factor XIII ${ }_{a^{\prime}}$ que a su vez se forma por acción de la trombina ${ }^{12,3,4,5,6,7,8,9}$.

\section{Regulación y modulación de la cascada}

La cascada de coagulación se va amplificando y acelerando, pero existe un mecanismo de "freno", porque sin control traería un taponamiento masivo, participa:

1. El flujo sanguíneo, arrastra factores activados, diluye su acción e impide acelerarse, por ello la estasis favorece la formación de trombos. 
2. El hígado actúa de filtro extrayendo factores activados o inactivándolos.

3. Existen proteasas que degradan o inhiben a ciertos factores activados.

Proteína C. Proenzima, se sintetiza en hígado, depende de vitamina $K$, es activada por la trombina. La $\mathrm{C}_{\mathrm{a}}$ degrada los factores $\mathrm{V}_{\mathrm{a}}$ y VIII $\mathrm{a}^{\prime}$ y limita la cascada. La trombina tiene triple papel: cataliza la formación de fibrina, activa a la enzima responsable de su entrecruzamiento, y una vez en marcha el proceso de coagulación y estabilización del coágulo tiende a limitarlo.

Antitrombina III. Sintetizada en hígado no depende de vitamina $K$, principal inhibidora de la coagulación, inhibe irreversiblemente a la trombina, la calicreína y los factores $\mathrm{IX}_{a^{\prime}} \mathrm{X}_{\mathrm{a}^{\prime}} \mathrm{XI}_{\mathrm{a}}$ y XII . La acción se incrementa por heparina, se encuentra en el endotelio de los vasos sanguíneos y en los gránulos de las células cebadas, facilita la unión de antitrombina III con factores procoagulantes activos. Existen anti-proteasas sanguíneas que ejercen acción anticoagulante pero menos potente como la

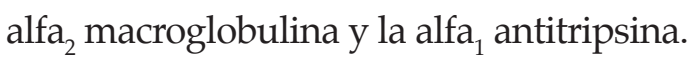

\section{Pruebas de coagulación sanguínea y las interpretaciones}

Tiempo de sangría. Evalúa las respuestas vascular y de plaquetas, ante una lesión vascular. Determina el tiempo de sangrado con el uso de un manguito de esfigmomanómetro en el antebrazo, a $40 \mathrm{mmHg}$. Se realiza una incisión superficial en antebrazo y se ve el tiempo en detener el sangrado, 1-9 minutos, si es mayor a 15 , indica un defecto en la respuesta inicial o se pincha sobre yema del dedo o lóbulo de la oreja, cesa la hemorragia en 1-3 minutos. El tiempo se prolonga por defecto en función plaquetaria, por trastornos vasculares, y enfermedad de Von
Willebrand, en trombocitopenia, coagulación Intravascular diseminada (CID), síndrome de Bernard-Soulier, enfermedad de Glanzmann, síndrome de Cushing, Hepatopatía severa y crónica, leucemia e insuficiencia de la médula ósea.

Tiempo de protrombina (TP), se prolonga por defectos en fibrinógeno, protrombina, y factores V, VII, y X, normal 11.0-12.5 segundos, más de 20 indica déficit en la coagulación. El cociente internacional normalizado (INR) resulta de dividir el tiempo de coagulación de los pacientes por la de un estándar conocido, normal 0,8-1,2. Se utiliza para regular dosis de warfarina, ver la presencia de hepatopatía crónica y el estado de la vitamina $\mathrm{K}$.

Tiempo de tromboplastina parcial (TTP), analiza la vía intrínseca, evalúa fibrinógeno, protrombina, y factores V, VIII, IX, X, XI y XII, su defecto origina una prolongación. Normal de 60-70 segundos. Evalúa la terapia con heparina. $\mathrm{Su}$ prolongación en deficiencia de los factores de coagulación anteriores, deficiencia de vitamina K, enfermedad hepática, CID, enfermedad de Von Willebrand, leucemia, hemofilia, y la administración de heparina.

\section{Trastornos congénitos de la coagulación}

Hemofilia A. La clásica por déficit del factor VIII, ligada al cromosoma $\mathrm{X}$, existen formas severas, moderadas y leves, frecuencia de 1:5, o 10,000 en población, abierta, Sufren de hemorragia muscular y de articulaciones, equimosis fáciles y sangrado prolongado luego de una lesión. El tratamiento, infusión de concentrados del factor VIII preparado de plasma humano o por DNA recombinante.

Hemofilia B. Deficiencias o disminución de factor IX. Prevalencia 1/10 del de la hemofilia A, 
tiempo de coagulación prolongado, existen formas severas, moderadas y leves.

Desórdenes del Fibrinógeno. Existen asociados a factores de riesgo cardiovascular: enfermedad arterial coronaria y periferica, diabetes, hipertensión, dislipidemias. Se eleva con el embarazo, la menopausia, el uso de hormonales y el tabaquismo. Las formas hereditarias son: la afibrinogenemia (deficiencia total, normal 250-350mg/dL), caracterizada por un cordón umbilical hemorrágico, equimosis, hemorragia de mucosas, hemorragia interna y abortos recurrentes, es autosómica recesiva. Hipofibrinogenemia niveles bajos, menor a $100 \mathrm{mg} / \mathrm{dL}$, adquirida o heredada. Síntomas similares, pero menos severos que en la afibrinogenemia. Las disfibrinogenemias son heterogéneas, afectan cualquier función del fibrinógeno. Datos clínicos: hemorragia, abortos espontáneos y el tromboembolismo.

Deficiencia de factor X111. cataliza la unión de los monómeros de fibrina. Las deficiencias hereditarias son autosómicas recesivas. La manifestación clínica: sangrado retrasado, la hemostasis primaria es normal, cordón umbilical sangrante, hemorragia intracraneal y hematomas del tejido blando.

Enfermedad de von Willebrand. Por déficit del factor von Willebrand (vWF), es la coagulopatia más común 8000 por millón, déficit de la adhesión plaquetaria y del factor VIII, causa sangrado similar al de una disfunción plaquetaria ó la hemofilia, ocurre en 125 por millón de personas. El tipo I, el más común, autosómico dominante con déficit cuantitativo de los multímeros del vWF. La tipo II se subdivide dependiendo de que si la proteína disfuncional ha disminuido ó aumentado su función durante su unión a las plaquetas. La tipo III es clínicamente severa y cursa con ausencia virtual del vWF, es heredada de manera recesiva.

\section{El Factor XI y la Activación por Contacto.} Cuando la sangre contacta superficies de carga negativa, se inician interacciones que incluyen al factor XI, precalicreína y el Quininógeno, que conllevan a la coagulación sanguínea, activación por contacto. Un déficit del factor XI da tendencia a sangrar relacionada con una lesión, es común en los Judíos Ashkenazic, se hereda en forma autosómica con homocigosis o heterocigosis compuesta.

Deficiencia en la Antitrombina. Sirve para inhibir a la trombina, el factor IX $X_{a}$ y el factor $X_{a}$. Afecta la síntesis o estabilidad de la antitrombina o los sitios de unión y/o la heparina en la antitrombina. La heparina aumenta su actividad 1000 veces más. Presente en el 2\% de los pacientes con enfermedad venosa tromboembólica, herencia autosómico dominante. La sintomática en 1 de 2000 a 1 de 5000 en la población. Las manifestaciones clínicas: trombosis en venas profundas y embolismo pulmonar, la trombosis arterial no es común, ocurre espontáneamente o en asociación a cirugía, trauma o embarazo. El tratamiento de los episodios agudos, infusión de heparina (por 5 a 7 días) seguida de terapia anticoagulante oral ${ }^{10,11,12,13,14,15}$

\section{Comentario}

La hemostasia es un mecanismo de defensa cuyo objetivo es evitar la pérdida de sangre y conservar la integridad vascular. Para el odontólogo el ver un paciente con historia de hematomas y hemorragias es frecuente por ello debe de tener la capacidad de hacer un diagnóstico y un tratamiento adecuado. La prevención y el conocimiento de las patologías que lo originan en este caso las coagulopatías congénitas es la 
mejor forma de evitar complicaciones hemorrágicas posterior a procedimientos quirúrgicos bucales.

El odontólogo debe tener conocimientos básicos, para tratar a pacientes con alteraciones de la hemostasia y poder realizar un correcto diagnóstico y un plan de tratamiento adecuado, tratamiento específico para cada trastorno de la hemostasia.

Antes de cualquier tratamiento, en caso de conocerse la patología, comentar con el hematólogo que trata al paciente.

\section{Referencias}

1. Catalano P. Trastornos hemostáticos. En: Roce L, Kaye D, editores. Medicina Interna en Odontología. 1 ed. Barcelona: Salvat; 1992. p. 431-65

2. Bermudo Añino L, Gutierres JL. Manejo del paciente con trastornos hemorrágicos. Tratamiento del paciente odontológico con hemopatías. En: Bermudo Añino L, Palma Gómez de la casa A, editores. Madrid: Glaxo-Smith-Liline Intigraf; 2001. p. 39-54.

3. Furie B, Furie BC. Mechanisms of thrombus formation. N Engl J Med 2008;359:938-949.

4. Furie B, Furie BC. Molecular basis of blood coagulation. En: Hematology.Basic principles and practice . 5th Edition. Hoffman R et al (eds). Churchill Livingstone Elsevier, Philadelphia, USA 2009; pp1819-1836.

5. Macfarlane RG. An enzyme cascade in the blood clotting mechanism, and its function as a biological amplifier. Nature 1964;202:498-499.

6. Davie EW, Ratnoff OD. Waterfall sequence for intrinsic blood clotting. Science 1964; 145: 1310-1312.

7. Hoffman M. Monroe DM. A cell-based model of hemostasis. Thromb Haemost 2001;85:958-965.

8. Hoffman M, Monroe DM. Rethinking the coagulation cascade. Curr Hematol Rep 2005;4: 391-396.9. Furie B, Furie BC. Thrombus formation in vivo. J Clin Invest. 2005;115:3355-62

10. Gómez K, McVey JH. Tissue factor initiated blood coagulation. Front.Biosci. 2006;11:1349-59

11. Páramo JA Monedero P, Hidalgo F, Hernández M. Fundamentos básicos para el empleo de hemoderivados y estrategias de ahorro de sangre en cirugía. Rev Med Univ Navarra 2008;52:9-14.

12. Sorensen B, Ingerslev J. Tailoring haemostatic treatment to patient requirements - an update on monitoring haemostatic response using thrombelastography. Haemophilia. 2005 ;11 Suppl 1:1-6.

13. Páramo JA. Coagulación intravascular diseminada. Med Clin (Barc) 2006;127:785-789.

14. Tieu BH, Holcomb JB, Schreiber MA. Coagulopathy: its pathophysiology and treatment in the injured patient. World J Surg. 2007;31:1055-64.

15. Lecumberri R, Páramo JA, Hidalgo F, Feliu J, Iglesias R, Rocha E. Reducción de las necesidades transfusionales en hemorragias adquiridas graves mediante factor VII activo recombinante. Med Clin (Barc) 2005;125:252-253.

Recepción: 02- 06-2012

Aceptado: 18-10-2012

Juan Manuel-Cortes-Ramírez Cardiólogo internista (doc_juancard52@hotmail.com)

Dirección: Guerrero \#128 Colonia Centro Zacatecas Zac. tel. 9229225

Traducción: Fabián Calixto Fraiz 